\title{
Symmetric Periodic Solutions of the Anisotropic Manev Problem
}

\author{
Manuele Santoprete ${ }^{\text {a) }}$ \\ Department of Mathematics and Statistics \\ University of Victoria, P.O. Box 3045 Victoria B.C., \\ Canada, V8W 3P4
}

\begin{abstract}
We consider the Manev potential in an anisotropic space, i.e., such that the force acts differently in each direction. Using a generalization of the Poincaré continuation method we study the existence of periodic solutions for weak anisotropy. In particular we find that the symmetric periodic orbits of the Manev system are perturbed to periodic orbits in the anisotropic problem.
\end{abstract}

PACS(2001): 05.45.-a, 45.50.Jf, 45.50.Pk

\footnotetext{
a)Electronic mail: msantopr@math.uvic.ca
} 


\section{Introduction}

In this paper we consider the Anisotropic Manev Problem (AMP) that was introduced by Diacu ${ }^{1}$ in the early 1990s. The work on the AMP was inspired by the Anisotropic Kepler Problem introduced by Gutzwiller in the early 1970s. Gutzwiller aimed to find connections between classical and quantum mechanics. His interest was stimulated by an old unsolved quantum mechanical problem formulated in a paper written by Einstein: ${ }^{2}$ even if the Born-Sommerfeld-Einstein condition (e.g. see Ref. 2) were appropriate to describe the semi-classical limit of quantum theory it was unclear how to find a classical approximation for nonintegrable systems.

Similarly the main reason for considering the AMP is to further analyze similarities between classical mechanics and quantum theory. Moreover, as it was remarked in Ref. 1, the AMP also brings general relativity into the game, since, the Manev's potential, explains the perihelion advance of the inner planets with the same accuracy as general relativity. ${ }^{3}$ It should be remarked that bringing general relativity into the game is of particular importance since a satisfactory quantum theory of gravitation does not exist.

Some of the qualitative features of the Anisotropic Manev Problem have already been studied. In Ref. 1, a large class of capture-collision and ejectionescape solutions is studied by means of the collision and infinity manifold techniques. In particular that paper also brought arguments favouring the chaoticity and nonintegrability of the system by showing the existence of heteroclinic orbits within the zero energy manifold. In Ref. 4 the occurrence of chaos on the zero energy manifold and the nonintegrability are finally proved, putting into evidence that the AMP is a very complex problem.

In this work, to gain a better understanding of the complicated dynamics of the AMP, we find the symmetric periodic orbits. Analyzing those orbits is especially important since, by now, it is well known that studying periodic orbits is a valuable general approach to tackle complex problems in classical mechanics. The existence of periodic orbits for small values of the anisotropy is proved using generalizations of the Poincaré continuation method developed in Refs. 5-7. 
The (planar) anisotropic Manev problem is described by the Hamiltonian

$$
H=\frac{1}{2} \mathbf{p}^{2}-\frac{1}{\sqrt{x^{2}+\mu y^{2}}}-\frac{b}{x^{2}+\mu y^{2}} .
$$

where $\mu>1$ is a constant and $\mathbf{q}=(x, y)$ is the position of one body with respect to the other considered fixed at the origin of the coordinate system, and $\mathbf{p}=\left(p_{x}, p_{y}\right)$ is the momentum of the moving particle. The constant $\mu$ measures the strength of the anisotropy and for $\mu=1$ we recover the classical Manev problem. Furthermore the equation of motion can be expressed as

$$
\left\{\begin{array}{l}
\dot{\mathbf{q}}=\mathbf{p} \\
\dot{\mathbf{p}}=-\frac{\partial H}{\partial \mathbf{q}}
\end{array} .\right.
$$

Now consider weak anisotropies, i.e choose the parameter $\mu>1$ close to 1 . Introducing the notation $r=\sqrt{x^{2}+y^{2}}$ and $\epsilon=\mu-1$ with $\epsilon \ll 1$ we can expand the Hamiltonian (1) in powers of $\epsilon$ and obtain

$$
H=\frac{1}{2} \mathbf{p}^{2}-\frac{1}{r}-\frac{b}{r^{2}}+\epsilon\left(\frac{1}{2 r}+\frac{b}{r^{2}}\right) \cos ^{2} \theta \equiv H_{0}+\epsilon W(r, \theta) .
$$

It should be pointed out that the term $W(r, \theta)$ becomes unbounded as $r \rightarrow 0$ so that a perturbation analysis is not correct on the ejection-collision orbits. This means that the global dynamics of the AMP cannot be completely described by perturbations to the Manev problem even at the limit $\epsilon \rightarrow 0$. However many interesting results concerning the Hamiltonian (11) for weak anisotropies (i.e. $\epsilon \ll 1$ ) can be found studying the Hamiltonian (3), some of which are presented in this paper.

In the next section we describe the symmetries of the AMP and we find some properties that will be useful to find symmetric periodic orbits. In Section III we prove a continuation theorem for the symmetric periodic orbits of "second kind", i.e. the non-circular ones. In Section IV we prove a continuation theorem for the orbits of "first kind", i.e. the circular ones, following the method developed in Ref. 8.

\section{Symmetries of the Anisotropic Manev Problem}

To find periodic orbits in the anisotropic problem it is peculiarly important to know the symmetries of the system, as it was, for example observed in Refs. 
5,6 . The symmetries of the problem under discussion have been examined in Ref. 1 and they are the same as the ones found in Ref. 7 for the anisotropic Kepler problem:

$$
\begin{aligned}
& E:\left(x, y, p_{x}, p_{y}, t\right) \longrightarrow\left(x, y, p_{x}, p_{y}, t\right) \\
& S_{0}:\left(x, y, p_{x}, p_{y}, t\right) \longrightarrow\left(x, y,-p_{x},-p_{y},-t\right) \\
& S_{1}:\left(x, y, p_{x}, p_{y}, t\right) \longrightarrow\left(x,-y,-p_{x}, p_{y},-t\right) \\
& S_{2}:\left(x, y, p_{x}, p_{y}, t\right) \longrightarrow\left(-x, y, p_{x},-p_{y},-t\right) \\
& S_{3}:\left(x, y, p_{x}, p_{y}, t\right) \longrightarrow\left(-x,-y,-p_{x},-p_{y}, t\right) \\
& S_{4}:\left(x, y, p_{x}, p_{y}, t\right) \longrightarrow\left(-x, y,-p_{x}, p_{y}, t\right) \\
& S_{5}:\left(x, y, p_{x}, p_{y}, t\right) \longrightarrow\left(x,-y, p_{x},-p_{y}, t\right) \\
& S_{6}:\left(x, y, p_{x}, p_{y}, t\right) \longrightarrow\left(-x,-y, p_{x}, p_{y},-t\right)
\end{aligned}
$$

where $E$ is the identity.

The symmetries above can be interpreted in the following way: let $\gamma(t)$ be a solution of (2), then $S_{i}(\gamma(t))$ is another solution for $i \in\{0,1,2,3,4,5,6\}$. For $i \in\{0,1,2,3,4,5,6\}$ the orbit $\gamma(t)$ will be called symmetric if and only if $S_{i}(\gamma(t))=\gamma(t)$.

Let us remark that the symmetries in (丑), together with the composition of functions, denoted by $\circ$, form an abelian group in which the operation acts according to the table below.

\begin{tabular}{c|cccccccc}
$\circ$ & $E$ & $S_{0}$ & $S_{1}$ & $S_{2}$ & $S_{3}$ & $S_{4}$ & $S_{5}$ & $S_{6}$ \\
\hline$E$ & $E$ & $S_{0}$ & $S_{1}$ & $S_{2}$ & $S_{3}$ & $S_{4}$ & $S_{5}$ & $S_{6}$ \\
$S_{0}$ & $S_{0}$ & $E$ & $S_{5}$ & $S_{4}$ & $S_{6}$ & $S_{2}$ & $S_{1}$ & $S_{3}$ \\
$S_{1}$ & $S_{1}$ & $S_{5}$ & $E$ & $S_{3}$ & $S_{2}$ & $S_{6}$ & $S_{0}$ & $S_{4}$ \\
$S_{2}$ & $S_{2}$ & $S_{4}$ & $S_{3}$ & $E$ & $S_{1}$ & $S_{0}$ & $S_{6}$ & $S_{5}$ \\
$S_{3}$ & $S_{3}$ & $S_{6}$ & $S_{2}$ & $S_{1}$ & $E$ & $S_{5}$ & $S_{4}$ & $S_{0}$ \\
$S_{4}$ & $S_{4}$ & $S_{2}$ & $S_{6}$ & $S_{0}$ & $S_{5}$ & $E$ & $S_{3}$ & $S_{1}$ \\
$S_{5}$ & $S_{5}$ & $S_{1}$ & $S_{0}$ & $S_{6}$ & $S_{4}$ & $S_{3}$ & $E$ & $S_{2}$ \\
$S_{6}$ & $S_{6}$ & $S_{3}$ & $S_{4}$ & $S_{5}$ & $S_{0}$ & $S_{1}$ & $S_{2}$ & $E$
\end{tabular}

From the table above it is easy to deduce the following

Proposition 1 The symmetries of the Anisotropic Manev Problem form an elementary abelian group of order eight, i.e. a group isomorphic to $\mathbf{Z}_{2} \times \mathbf{Z}_{2} \times$ $\mathbf{Z}_{2}$.

The symmetries in (4), (except $E$ and $S_{6}$ ) are very useful to find symmetric periodic orbits, especially by means of the continuation method, as we show 
in the next two sections. Some important properties of the symmetric orbit, summarized in Ref. 7 , are expressed in the following lemma:

Lemma 1 (i) For $i=1$ (resp. $i=2$ ) we have that an orbit $\gamma(t)$ is $S_{i^{-}}$ symmetric if and only if it crosses the $x$ axis (resp. $y$ axis) orthogonally.

(ii) An orbit $\gamma(t)$ is $S_{0}$-symmetric if and only if it has a point on the zero velocity curve.

(iii) For $i=4,5$ an orbit $\gamma(t)$ is $S_{i}$-symmetric if and only if it is $S_{0^{-}}$ symmetric.

(iv) All the $S_{3}$-symmetric periodic orbits are periodic.

The properties of the $S_{i}$-symmetric orbits were first studied by Birkhoff ${ }^{9}$ for the restricted three body problem and later by many other authors. In particular Casasayas and Llibre ${ }^{7}$ state a proposition that gives a technique useful to obtain symmetric periodic orbits with respect to $S_{0}, S_{1}, S_{2}$ for the anisotropic Kepler problem that are verified also for the problem under discussion in this paper:

Proposition 2 (i) For $i=1$ (resp. $i=2$ ) we have that an orbit $\gamma(t)$ is a $S_{i}$-symmetric periodic orbit if and only if it crosses the $x$ axis (resp. $y$ axis) orthogonally at two distinct points.

(ii) An orbit $\gamma(t)$ is a $S_{0}$-symmetric periodic orbit if and only if it meets the zero velocity curves at two distinct points.

(iii) An orbit $\gamma(t)$ is a $S_{1}$ and $S_{2}$ symmetric periodic orbit if and only if it crosses the $x$ axis and the $y$ axis orthogonally.

(iv) For $i=1,2$ an orbit $\gamma(t)$ is a $S_{0}$ and $S_{i}$-symmetric periodic orbit if and only if it meets the zero velocity curve and crosses the $x$, respectively $y$ axis orthogonally.

(v) For $i=4,5$, if an orbit $\gamma(t)$ is $S_{i}$-symmetric then it is $S_{0}$-symmetric and periodic.

Now we want to find the symmetric periodic orbit for the unperturbed problem $(\epsilon=0$ or $\mu=1)$ and continue them to periodic solutions of the anisotropic system (for $\epsilon \ll 1$ ). Firstly we observe that, by Proposition 2, 
the $S_{i}$ symmetric orbits with $i=0,4,5$ must meet the zero velocity curve at two points, i.e. there must be a point where $K=0$, but since $K$ is a constant of motion it must be zero along the orbit. Therefore such orbits are ejection-collision orbits, are not periodic and cannot be studied by means of the continuation method. Hence we are going to consider the symmetric periodic orbit with $i=1,2$, and also the ones with $i=3$ that are the circular orbits of the unperturbed problem.

To exploit those properties of the symmetric periodic orbits it is convenient to write the equation of motion in different coordinates.

For the $S_{i}$ symmetric orbits with $i=1,2$, as it was noted in Ref. 5 , it is convenient to write the canonical equations of the restricted three body problem using the Delaunay variables in the rotating frame. ${ }^{5}$ Also the Poincaré synodic variables can be used to find symmetric periodic orbits of the restricted three body problem. ${ }^{6}$ The anisotropic Manev problem is different since the Hamiltonian that describes it is time independent, hence the idea of using rotating coordinates in the present case cannot be applied. Moreover our problem is nondegenerate, however even in our case it is advantageous to perform a change of variables and apply a variation of the action angle variables used in Refs. 4, 12. Here the nondegeneracy of the problem plays a role similar to the rotating coordinate system in the restricted three body problem.

For the $S_{3}$ symmetric orbits we can instead consider the equations in the rotating frame, and prove a theorem similar to the one proved in Ref. 8 for the anisotropic Kepler problem (in Ref. 8 the author remarks that the analysis of the Kepler problem can be redone in the Manev case, but he doesn't provide a proof) .

\section{The $\mathrm{S}_{\mathrm{i}}$ Symmetric Orbits with $\mathrm{i}=1,2$}

We recall that the action variables introduced in Refs. 4, 12 are given by

$$
\left\{\begin{array}{l}
I=\frac{1}{2 \pi} \oint p_{r} d r=-\sqrt{K^{2}-2 b}+\frac{1}{2} \sqrt{\frac{2}{|h|}} \\
K=q_{1} p_{2}-q_{2} p_{1}
\end{array}\right.
$$

where $h$ is the energy constant and $K$ is the angular momentum. These variables are defined for $h<0$ and $K^{2}>2 b, I>0$, to avoid collision orbits 
as well as circular orbits. The related frequencies are

$$
\left\{\begin{array}{l}
\omega_{I}=\frac{1}{\left(I+\sqrt{K^{2}-2 b}\right)^{3}} \\
\omega_{K}=\frac{K}{\sqrt{K^{2}-2 b}\left(I+\sqrt{K^{2}-2 b}\right)^{3}}
\end{array}\right.
$$

and $\theta$ and $\phi$ are the angle variables associated to $K$ and $I$ respectively. The unperturbed Hamiltonian in the new variables can be written as

$$
H_{0}=-\frac{1}{2\left(I+\sqrt{K^{2}-2 b}\right)^{2}} .
$$

Now we can consider new variables that are linear combination of the previous ones. They are defined by the following canonical transformation

$$
\left\{\begin{array}{l}
L=K+I \\
G=-I \\
l=\theta \\
g=\theta-\phi
\end{array}\right.
$$

Where $l$ is the mean anomaly (where $l(t)=\omega_{L}\left(t-t_{0}\right)$ and $t_{0}$ is the time of pericenter passage), $g$ is the longitude of pericenter as they are defined for the Manev problem in Ref. 13. Moreover also the action variables can be written in terms of the orbital elements of the Manev problem. If we set

$$
a=\frac{1}{2|h|} \quad \text { and } \quad e=\sqrt{1-2\left(K^{2}-2 b\right)|h|}
$$

as in Refs. 4, 13 then

$$
G=-a^{1 / 2}\left[1-\left(1-e^{2}\right)^{1 / 2}\right] \quad \text { and } \quad L=-G \pm \sqrt{a\left(1-e^{2}\right)+2 b}
$$

where $a$ is the pseudo-semimajor axis, $e$ is the pseudo-eccentricity, and the sign + (resp. -) holds for $K>0$ (resp. <0). The conditions to avoid collision orbits and circular orbits, on which $g$ becomes meaningless, can be written in terms of the orbital elements as $a>0$ and $0<e<1$. The new unperturbed Hamiltonian is

$$
H_{0}=-\frac{1}{2\left(-G+\sqrt{(G+L)^{2}-2 b}\right)^{2}}
$$


so the perturbed equations of motion become

$$
\left\{\begin{array}{l}
\dot{L}=-\frac{\partial\left(H_{0}+\epsilon W\right)}{\partial l}=-\epsilon \frac{\partial W}{\partial l} \\
\dot{G}=-\frac{\partial\left(H_{0}+\epsilon W\right)}{\partial g}=-\epsilon \frac{\partial W}{\partial g} \\
\dot{l}=\frac{\partial\left(H_{0}+\epsilon W\right)}{\partial L}=\omega_{L}+\epsilon \frac{\partial W}{\partial L} \\
\dot{g}=\frac{\partial\left(H_{0}+\epsilon W\right)}{\partial G}=\omega_{G}+\epsilon \frac{\partial W}{\partial G}
\end{array}\right.
$$

where $W$ is expressed in the new variables and

$$
\left\{\begin{array}{l}
\omega_{L}=\omega_{K}=\frac{G+L}{\left(-G+\sqrt{(G+L)^{2}-2 b}\right)^{3} \sqrt{(G+L)^{2}-2 b}} \\
\omega_{G}=\omega_{K}-\omega_{I}=\frac{G+L-\sqrt{(G+L)^{2}-2 b}}{\left(-G+\sqrt{(G+L)^{2}-2 b}\right)^{3} \sqrt{(G+L)^{2}-2 b}}
\end{array}\right.
$$

With these preparations, i.e. the introduction of the action angle variables (6), we are well on our way to estabilishing the following result:

Theorem 1 Let $\gamma(t)$ be an $S_{i}$-symmetric periodic orbit of the Manev problem with $i=1,2$. Let the period be $\tau$ and set $\epsilon=\mu-1$ with $\epsilon \ll 1$. Then there exists a $\tau$-periodic solution of the Anisotropic Manev problem $\gamma_{\epsilon}(t)$ such that $\gamma_{\epsilon}(t)=\gamma(t)+O(\epsilon)$.

PROOF: Let's consider an $S_{1}$ symmetric orbit of period $\tau=2 \pi m / k(m, k$ relatively prime integers). We remark that, since the equations of motion are autonomous, we can reduce to study the symmetric orbits that have either the pericenter or the apocenter on the positive $x$ axis at $t=0$.

If at $t=0, \epsilon=0$, the pericenter of this orbit is on the positive $x$ axis, and it is crossing the $x$ axis perpendicularly, we have

$$
g(0)=0 \quad \text { and } \quad l(0)=0 .
$$

Since the periodic orbit is $S_{1}$ symmetric, by Proposition 2, at the half period one has

$$
g(\tau / 2)=m \pi \quad l(\tau / 2)=k \pi
$$

that follows from the solution of (8) for $\epsilon=0$ :

$$
\begin{array}{ll}
L=\text { const. } & G=\text { const. } \\
l=\omega_{L} t & g=\omega_{G} t
\end{array}
$$


Now if, for $\epsilon \neq 0$ we consider only $S_{1}$ symmetric solutions of (\$), it follows from the implicit function theorem that if the functional determinant

$$
D=\operatorname{det}\left(\begin{array}{cc}
\partial l / \partial L & \partial l / \partial G \\
\partial g / \partial L & \partial g / \partial G
\end{array}\right) \neq 0
$$

at

$$
t=\tau / 2 \quad \epsilon=0
$$

then (10) would be satisfied for $\epsilon>0$. To compute the determinant we can by analyticity substitute (10) into (11) to find out at the time $t=\tau / 2$ that

$$
D=\frac{6 b(\tau / 2)^{2}}{\left(-G+\sqrt{(G+L)^{2}-2 b}\right)^{7}\left((G+L)^{2}-2 b\right)^{3 / 2}} \neq 0
$$

Thus the existence of $S_{1}$ symmetric periodic orbits of period $\tau$ obtained from the $\tau$ periodic $S_{1}$ symmetric solutions of the unperturbed problem, that at $t=0$ have the pericenter on the positive $x$ axis, is readily established.

On the other hand, if at $t=0, \epsilon=0$, the apocenter is on the positive $x$ axis, and it is crossing the $x$ axis perpendicularly, we have

$$
g(0)=\pi / \lambda \text { and } l(0)=-\pi / \lambda
$$

where $\lambda=\left(\omega_{L}-\omega_{G}\right) / \omega_{L}$. By Proposition 1 , at the half period we have

$$
g(\tau / 2)=(m+1 / \lambda) \pi \quad l(\tau / 2)=(-1 / \lambda+k) \pi .
$$

Instead of computing the functional determinant directly, in this case, it is easier to consider the new variables given by the relations,

$$
\left\{\begin{array}{l}
\tilde{L}=L \\
\tilde{G}=G \\
\tilde{l}=l+\pi / \lambda_{0} \\
\tilde{g}=g-\pi / \lambda_{0}
\end{array}\right.
$$

that define a family of canonical transformations parametrized by $\lambda_{0}\left(L_{0}, G_{0}\right)$. For each orbit choose a different transformation from the family (17), where $\lambda_{0}=\lambda$ is a fixed quantity defined by the value of the action variables along the periodic orbit under consideration.

The equations (16), expressed in the new variables, are of the same form as in (10). Thus the functional determinant, in the new variables, is exactly $D$, and the existence of the remaining $S_{1}$-symmetric $\tau$-periodic orbits follows. 
Now the proof for the $S_{2}$-symmetric orbits can be done along the same lines. Consider an $S_{2}$ symmetric periodic orbit of period $\tau=2 \pi m / k$. If at $t=0$, $\epsilon=0$ the pericenter of the orbit is on the positive $y$ axis and it is crossing the $y$ axis perpendicularly, we have

$$
g(0)=\pi / 2 \quad \text { and } \quad l(0)=0
$$

Since the periodic orbit is $S_{2}$ symmetric one has, at the half period

$$
g(\tau / 2)=m \pi+\pi / 2 \quad l(\tau / 2)=k \pi .
$$

Now we consider only $S_{2}$ symmetric solutions of (8) for $\epsilon \neq 0$ again it follows from the implicit function theorem that if the determinant $D$ computed at $t=\tau / 2$ for $\epsilon=0$ is non zero then (19) would be satisfied for $\epsilon>0$. It is trivial to see from (14) that $D \neq 0$, and hence we found $S_{2}$ symmetric periodic orbits for the perturbed problem.

For the $S_{2}$-symmetric orbits having the apocenter on the positive $x$ axis at $t=0$ the canonical transformation (17) can be used. Again we find the same expression for the functional determinant and hence, by the implicit function theorem, the existence of the remaining $S_{2}$-symmetric periodic orbits is proved.

It is interesting to remark that Theorem 1 and its proof can be easly extended to consider any $S_{i}$-symmetric perturbation with $i=1,2$ and a very general class of nondegenerate integrable Hamiltonians, however such a generalization is trivial and not strictly related to the problem under consideration and hence it will not be discussed any further.

We can also observe that for $b=0$, i.e. for the Kepler problem, the determinant in (14) is zero. Thus in the case of the Anisotropic Kepler Problem, the continuation theorem proved above cannot be applied, and the existence of symmetric periodic orbits of "second kind" (for weak anisotropies) remains unclear. On the other hand the continuation theorem that we prove in the next section (for the circular orbits) can be applied to the Anisotropic Kepler Problem ${ }^{8}$ and hence at least the existence of symmetric periodic orbits of the first kind is a well established fact.

\section{The $\mathrm{S}_{3}$ Symmetric Orbits}

Again we can consider the Anisotropic Manev Problem taking the parameter

$\mu$ close to 1 . Let the flow $\Phi(t,(\mathbf{r}, \dot{\mathbf{r}}), \mu)$ of the equation of motion (1). In this section we prove the following theorem: 
Theorem 2 Let $\mathbf{r}^{0}(t)$ be a $S_{3}$-symmetric periodic orbit of the Manev problem, i.e. a circular one. Set $\epsilon=\mu-1$, and let $\tau$ be the period of $\mathbf{r}^{0}(t)$. Then there exists a $\tau$-periodic solution $\Phi(t,(\mathbf{r}(\epsilon), \dot{\mathbf{r}}(\epsilon)), \epsilon)$ of the Anisotropic Manev problem such that $\Phi(t,(\mathbf{r}(0), \dot{\mathbf{r}}(0)), 0)=\left(\mathbf{r}^{0}(t), \dot{\mathbf{r}}^{0}(t)\right)$.

\section{A. The equation of motion}

Now using the same notation as in Ref. 8 let $\mathbf{r}^{\mathbf{0}}(\mathbf{t})$ be a circular solution of the Manev problem which correspond to $\mu=1$ in the $x y$-plane, $\omega$ its angular speed and $a$ its radius. For $\epsilon=\mu-1 \neq 0$ we set,

$$
\mathbf{r}(t, \epsilon)=\mathbf{r}^{0}(t)+\epsilon \mathbf{s}(t, \epsilon)
$$

Expanding $\nabla H$ in powers of $\mu-1$ sufficiently small, after substituting the expression for $\mathbf{r}$ given above, considering the notation $\mathbf{r}^{0}(t)=x_{0}(t)+i y_{0}(t)$ and $\mathbf{s}=u+i v$ we have that $\mathbf{r}(t, \epsilon)$ is a solution of equation of motion defined by (1i) if, and only if, $\mathbf{s}(t, \epsilon)$ is a solution of the equations

$$
\begin{aligned}
& \ddot{u}=-\left(\frac{1}{a^{3}}-\frac{3 x_{0}^{2}}{a^{5}}-\frac{8 b x_{0}^{2}}{a^{6}}+\frac{2 b}{a^{4}}\right) u+\left(\frac{3 x_{0} y_{0}}{a^{5}}+\frac{8 b x_{0} y_{0}}{a^{6}}\right) v+\eta(t)+O(\epsilon) \\
& \ddot{v}=\left(\frac{3 x_{0} y_{0}}{a^{5}}+\frac{8 b x_{0} y_{0}}{a^{6}}\right) u-\left(\frac{1}{a^{3}}-\frac{8 b y_{0}^{2}}{a^{6}}-\frac{3 y_{0}^{2}}{a^{5}}+\frac{2 b}{a^{4}}\right) v+\xi(t)+O(\epsilon)
\end{aligned}
$$

where

$$
\begin{aligned}
& \eta(t)=\frac{3 x_{0} y_{0}^{2}}{a^{5}}+\frac{4 b x_{0} y_{0}^{2}}{a^{6}} \\
& \xi(t)=\frac{3 y_{0}^{2}}{2 a^{5}}-\frac{y_{0}}{a^{3}}+\frac{4 b y_{0}^{3}}{a^{6}}-\frac{2 b y_{0}}{a^{4}}
\end{aligned}
$$

Consider the orthonormal frame in $\mathbf{R}^{2}, \mathbf{e}_{1}(t)$ and $\mathbf{e}_{2}(t)$ defined by

$$
\mathbf{e}_{1}=\frac{\mathbf{r}^{0}}{\left|\mathbf{r}^{0}\right|}=e^{i \omega t}=\cos \omega t+i \sin \omega t, \quad \mathbf{e}_{2}=i \mathbf{e}_{1}
$$

and using the same notation as in Ref. 8 where

$$
\mathbf{s}=x_{1} \mathbf{e}_{1}+x_{2} \mathbf{e}_{2}, \quad \dot{\mathbf{s}}=y_{1} \mathbf{e}_{1}+y_{2} \mathbf{e}_{2}
$$

equation (21) can be written in an equivalent form as:

$$
\dot{\mathbf{z}}=A_{0}(t)+A \mathbf{z}+O(\epsilon),
$$

where $\mathbf{z}=\left(x_{1}, x_{2}, y_{1}, y_{2}\right)^{T}$, and 


$$
A_{0}=\left(\begin{array}{c}
0 \\
0 \\
\alpha(t) \\
\beta(t)
\end{array}\right) \quad A=\left(\begin{array}{cccc}
0 & \omega & 1 & 0 \\
-\omega & 0 & 0 & 1 \\
2 \omega^{2}+2 \frac{b}{a^{4}} & 0 & 0 & \omega \\
0 & -\omega^{2} & -\omega & 0
\end{array}\right)
$$

where

$$
\begin{aligned}
& \alpha(t) \cos \omega t-\beta(t) \sin \omega t=\eta(t) \\
& \alpha(t) \sin \omega t+\beta(t) \cos \omega t=\xi(t)
\end{aligned}
$$

or equivalently,

$$
\begin{aligned}
& \alpha(t)=\sin ^{2} \omega t\left(\frac{1}{2 a^{2}}+\frac{2 b}{a^{3}}\right) \\
& \beta(t)=-\sin \omega t \cos \omega t\left(\frac{1}{a^{2}}+\frac{2 b}{a^{3}}\right)
\end{aligned}
$$

The eigenvalues of $A$ are 0 , with multiplicity two, $\frac{i}{a^{3 / 2}}$ and $-\frac{i}{a^{32}}$. One of the two eigenvalues vanishes because the system is autonomous, and the second due to the presence of the first integral $H$.

Now consider the real Jordan form $J$ of $A$. The matrix $J$ is defined by the relation $J=\mathcal{T}^{-1} A \mathcal{T}$ where $\mathcal{T}$ is

$\mathcal{T}=\left(\begin{array}{cccc}2 \omega^{2} a^{3} & 0 & \frac{\omega^{2} a^{4}+2 b}{a} & 0 \\ 0 & -\frac{\omega\left(3 \omega^{2} a^{4}+2 b\right)}{a} & 0 & -2 \frac{\omega a^{2}\left(\omega^{2} a^{4}+2 b\right)}{(a)^{3 / 2}} \\ 0 & \frac{1}{2} \frac{4 a\left(\omega^{2} a^{4}+b\right)+2\left(\omega^{2} a^{4}+2 b\right)^{2}}{a^{5}} & 0 & \frac{\left(\omega^{2} a^{4}+2 b\right)^{2}}{a^{7 / 2}} \\ -\frac{\omega\left(\omega^{2} a^{4}+2 b\right)}{a} & 0 & -\frac{\omega\left(\omega^{2} a^{4}+2 b\right)}{a} & 0\end{array}\right)$

and the columns of $\mathcal{T}$ are the generalized eigenvectors of $A$.

The vector $J_{0}=\mathcal{T}^{-1} A_{0}$ and the matrix $J$ are:

$$
J_{0}=\left(\begin{array}{c}
j_{1}(t) \\
j_{2}(t) \\
j_{3}(t) \\
j_{4}(t)
\end{array}\right), \quad J=\left(\begin{array}{cccc}
0 & 0 & 0 & 0 \\
1 & 0 & 0 & 0 \\
0 & 0 & 0 & \frac{\sqrt{a}}{a^{2}} \\
0 & 0 & -\frac{\sqrt{a}}{a^{2}} & 0
\end{array}\right)
$$

where the fact that $j_{1}(t)=\left(2 \omega^{3} a^{2}-\frac{\omega\left(\omega^{2} a^{4}+2 b\right)}{a^{2}}\right)^{-1} \beta(t)$ is the only information about $J_{0}$ that we need to retain. Furthermore we remark that $\omega^{2} a^{4}-a-2 b=$ 
0 gives the relation between $a$ and $\omega$ and solving this equations gives only one positive solution (for $b>0$ ).

Letting $\mathbf{z}=\mathcal{T} \zeta$, the equation of motion becomes

$$
\dot{\zeta}=J_{0}(t)+J \zeta+O(\epsilon)
$$

and its flow is given by

$$
\psi(t, \zeta, \epsilon)=\gamma(t)+e^{J t}+O(\epsilon)
$$

where by the variation of constants

$$
\gamma(t)=e^{J t} \int_{0}^{t} e^{-J s} J_{0}(s) d s
$$

Therefore we have

$$
e^{J t}=\left(\begin{array}{cccc}
1 & 0 & 0 & 0 \\
t & 1 & 0 & 0 \\
0 & 0 & \cos \frac{\sqrt{a}}{a^{2}} t & \sin \frac{\sqrt{a}}{a^{2}} t \\
0 & 0 & -\sin \frac{\sqrt{a}}{a^{2}} t & \cos \frac{\sqrt{a}}{a^{2}} t
\end{array}\right)
$$

and from (26) we obtain

$$
\gamma(t)=\left(\begin{array}{c}
\gamma_{1}(t) \\
\gamma_{2}(t) \\
\gamma_{3}(t) \\
\gamma_{4}(t)
\end{array}\right)
$$

where we retain only the information that

$$
\gamma_{1}(t)=\left(2 \omega^{3} a^{2}-\frac{\omega\left(\omega^{2} a^{4}+2 b\right)}{a^{2}}\right)^{-1} \int_{0}^{t} \beta(s) d s
$$

\section{B. The periodicity equation}

Since the Hamiltonian $H$ of the anisotropic Manev problem is $S_{3}$-symmetric, as we have shown, we can write the periodicity equation as $\mathrm{in}^{8}$,

$$
\Phi\left(\frac{\tau}{2},(\mathbf{r}, \dot{\mathbf{r}}), \epsilon\right)=-(\mathbf{r}, \dot{\mathbf{r}})
$$


Then it easy to check that $\Phi(t,(\mathbf{r}, \dot{\mathbf{r}}), \epsilon)$ is a periodic solution of the equation of motion with period $\tau$. To find periodic solutions we have to verify that (29) is satisfied for a family of initial conditions. Equation (29) in $\zeta$ coordinates is

$$
\psi\left(\frac{\tau}{2}, \zeta, \epsilon\right)-\zeta=0
$$

where $\psi(t, \zeta, \epsilon)$ is the flow of (24). Let us denote by $\mathcal{P}(\zeta, \epsilon)$ the left hand side of the periodicity equation (29), that is, let

$$
\mathcal{P}(\zeta, \epsilon)=\psi(\tau / 2, \zeta, \epsilon)-\zeta=\gamma(\tau / 2)+\left(e^{J \frac{\tau}{2}}-I\right)=0
$$

Using (25) we notice that the requirement

$$
\mathcal{P}\left(\zeta^{*}, 0\right)=\gamma(\tau / 2)+\left(e^{J \frac{\tau}{2}}-I\right) \zeta^{*}=0,
$$

imposes the restrictions

$$
\gamma_{1}(\tau / 2)=0 \quad \zeta_{1}^{*}=-\frac{2}{\tau} \gamma_{2}(\tau / 2) \quad \zeta_{2}^{*}=\text { arbitrary }
$$

and

$$
\begin{aligned}
& \zeta_{3}^{*}=\frac{1}{2\left(1-\cos \alpha^{*}\right)}\left(-\gamma_{3}(\tau / 2)\left(\cos \alpha^{*}-1\right)+\gamma_{4}(\tau / 2) \sin \alpha^{*}\right) \\
& \zeta_{4}^{*}=\frac{-1}{2\left(1-\cos \alpha^{*}\right)}\left(\gamma_{3}(\tau / 2) \sin \alpha^{*}+\gamma_{4}(\tau / 2)\left(\cos \alpha^{*}-1\right)\right)
\end{aligned}
$$

where $\alpha^{*}=\pi(1+2 b / a)^{-1 / 2}$. It easy to see from (23) and (28) that $\gamma_{1}(\tau / 2)=$ 0 , therefore, we take

$$
\zeta^{*}=\left(\zeta_{1}^{*}, \zeta_{2}^{*}, \zeta_{3}^{*}, \zeta_{4}^{*}\right)^{T}
$$

with $\zeta_{2}^{*}$ arbitrary, for the moment. Now using the flow (25), we determine that the Jacobian matrix of $\mathcal{P}$ with respect to the variables $\zeta$ evaluated at the point $\left(\zeta^{*}, 0\right)$ is given by

$$
\left(\begin{array}{cccc}
0 & 0 & 0 & 0 \\
\tau / 2 & 0 & 0 & 0 \\
0 & 0 & \cos \alpha^{*}-1 & \sin \alpha^{*} \\
0 & 0 & -\sin \alpha^{*} & \cos \alpha^{*}-1
\end{array}\right)
$$

Consider the system of three equations formed by those in (31) corresponding to the indices $\mathrm{i}=2,3,4$ and fix the variable $\zeta_{2}=\zeta_{2}^{*}$. Its Jacobian matrix has determinant $\tau\left(1-\cos \alpha^{*}\right)$, that is always positive since $0<\pi(1+2 b / a)^{-1 / 2} \leq \pi$. Therefore the implicit function theorem guarantees the existence of analytic 
functions $\zeta_{i}=\zeta_{i}(\epsilon), i=1,3,4$ in a neighborhood of $\epsilon=0$, satisfying the equations

$$
\mathcal{P}_{i}(\zeta, \epsilon)=0, \quad(i=2,3,4)
$$

where

$$
\zeta(\epsilon)=\left(\zeta_{1}(\epsilon), \zeta_{2}^{*}, \zeta_{3}(\epsilon), \zeta_{4}(\epsilon)\right)
$$

and such that

$$
\zeta_{i}(0)=\zeta_{i}^{*} \quad(i=1,2,3,4) .
$$

It remains to show, in order to have periodicity, that also the remaining equation

$$
\mathcal{P}_{1}(\zeta(\epsilon), \nu(\epsilon), \epsilon)=0,
$$

is satisfied in a, possibly smaller neighborhood of $\epsilon=0$. That will be done employing a first integral of the system under discussion, i.e. the Hamiltonian.

\section{Integral of motion}

Since the Hamiltonian is a integral of motion of the problem under discussion we can apply the same analysis as in Refs. 8, 14. In particular using the same notations as in Ref. 8 we can define

$$
H_{\epsilon}(\mathbf{z}, t)=H(\mathbf{r}, \dot{\mathbf{r}}, \epsilon)
$$

where $H_{\epsilon}(\mathbf{z}, t)$ is a time-dependent, $\tau$-periodic first integral for system (22). The above integral satisfies the following relation

$$
H_{\epsilon}(\mathbf{z}, t+\tau / 2)=H_{\epsilon}(\mathbf{z}, t)
$$

for all $t$, since $H(-\mathbf{r},-\dot{\mathbf{r}})=H(\mathbf{r}, \dot{\mathbf{r}}), \quad \mathbf{r}(t)=\mathbf{r}^{0}(t)+\epsilon \mathbf{s}(t)$ and

$$
\mathbf{r}^{0}(t+\tau / 2)=-\mathbf{r}^{0}(t) \quad, \quad \mathbf{s}(\mathbf{z}, t+\tau / 2)=-\mathbf{s}(\mathbf{z}, t) .
$$

Performing a change of coordinates we can define $\mathcal{H}_{\epsilon}(\zeta, t)=H_{\epsilon}(\mathcal{T} \zeta, t)$, hence (41) can be written as

$$
\mathcal{H}_{\epsilon}(\zeta, t+\tau / 2)=\mathcal{H}_{\epsilon}(\zeta, t)
$$

Moreover since $\mathcal{H}_{\epsilon}$ is an integral of motion it verifies that

$$
\mathcal{H}_{\epsilon}(\phi(\zeta, \epsilon, t))=H_{\epsilon}(\zeta, 0)
$$


Thus applying equations (42-43) it follows that

$$
\mathcal{H}_{\epsilon}(\psi(\tau / 2, \zeta, \epsilon), 0)=\mathcal{H}_{\epsilon}(\zeta, 0)
$$

and by means of the Mean Value Theorem we obtain

$$
\nabla_{\zeta} \mathcal{H}_{\epsilon}(\tilde{\zeta}, 0) \cdot \mathcal{P}(\zeta, \epsilon)=0
$$

where $\nabla_{\zeta} \mathcal{H}_{\epsilon}$ is the gradient of $\mathcal{H}_{\epsilon}$ with respect to $\zeta$, and $\tilde{\zeta}$ is a point on the segment joining $\zeta$ to $\psi(\tau / 2, \zeta, \epsilon)$.

Expanding $\Psi(\epsilon)=\psi(\tau / 2, \zeta, \epsilon)$ in power of $\epsilon$ sufficently small it is easy to show (see Ref. 8) that $\Psi(\epsilon)=\zeta^{*}+O(\epsilon)$ and consequently

$$
\tilde{\zeta}=s \zeta(\epsilon)+(1-s) \Psi(\epsilon)=\zeta^{*}+O(\epsilon)
$$

for some $s \in(0,1)$. Moreover if we also expand the Hamiltonian $H_{\epsilon}(\mathbf{z}, 0)$ in powers of $\epsilon$ we get

$$
H_{\epsilon}(\mathbf{z}, 0)=H_{0}+\epsilon\left(H_{1}+H_{2} \cdot \mathbf{z}\right)+O\left(\epsilon^{2}\right)
$$

or, in $\zeta$ coordinates

$$
\mathcal{H}_{\epsilon}(\zeta, 0)=\mathcal{H}_{0}+\epsilon\left(\mathcal{H}_{1}+\mathcal{H}_{2} \cdot \zeta\right)+O\left(\epsilon^{2}\right),
$$

where $\mathcal{H}_{0}=H_{0}=\left(\frac{1}{2} \omega^{2} a^{2}-\frac{1}{a}-\frac{b}{a^{2}}\right), \mathcal{H}_{1}=H_{1}$ and $\mathcal{H}_{2}=\mathcal{T}^{T} H_{2}=\mathcal{T}^{T}\left(a^{-2}+\right.$ $\left.2 b a^{-3}, 0,0, a \omega\right)=\left(a \omega^{2} \zeta_{1}, 0,0,0\right)$. Hence we obtain

$$
\frac{1}{\epsilon} \nabla_{\zeta} \mathcal{H}_{\epsilon}(\tilde{\zeta}, 0)=\mathcal{H}_{2}+O(\epsilon)
$$

With these preparations equation (44) reduces to the equation in the unknown $\mathcal{P}_{1}$

$$
\left[a \omega^{2}+O(\epsilon)\right] \mathcal{P}_{1}=0 .
$$

since, for small $\epsilon$, we already found in Sec. IV B that $\mathcal{P}_{i}=0$ for $i=2,3,4$. It is easy to see that for $\epsilon=0$ the equation above has solution $\mathcal{P}_{1}=0$. Thus, by continuity, $\left[a \omega^{2}+O(\epsilon)\right]$ is different from zero for $\epsilon$ sufficiently small. Therefore for such values of $\epsilon$ this equation has a unique solution that is the trivial one. Consequently the remaining equation

$$
\mathcal{P}_{1}(\zeta(\epsilon), \epsilon)=0
$$

is also satisfied in a possibly smaller neighborhood of $\epsilon=0$. Hence all the equations of the periodicity system (31) are satisfied when $\zeta=\zeta(\epsilon)$, as long as $\epsilon$ is sufficiently small. This completes the proof of Theorem 2 . 


\section{Acknowledgments}

The author is grateful to Professor Florin Diacu for his enlightening comments and suggestions. This work was supported by an University of Victoria Fellowship and a Howard E. Petch Research Scholarship.

\section{References}

${ }^{1}$ S. Craig, F. Diacu, E. A. Lacomba, E. Perez, "On the anisotropic Manev problem", J. Math. Phys., 40, 1359-1375 (1999)

${ }^{2}$ A. Einstein, "Zum Quantensatz von Sommerfeld und Epstein", Verhandl. Deutsch. Phys. Gesellsch., 19, 82-92 (1917)

${ }^{3}$ Y. Hagiara, Celestial Mechanics (MIT Press, Cambridge, MA, 1975), Vol. II part I

${ }^{4}$ F. Diacu, M. Santoprete, "Nonintegrability and Chaos in the Anisotropic Manev problem", Physica D, 156,39-52 (2001)

${ }^{5}$ R.B. Barrar, "Existence of periodic orbits of the second kind in the restricted problem of three bodies" , Astronomical J. 70,3-4 (1965)

${ }^{6}$ A. Milani, "Stability and Bifurcation of Symmetric Periodic Orbits", Rend. Circ. Mat. Palermo 34, 161-191 (1985)

7 J. Casasayas, J. Llibre, "Qualitative Analysis of the Anisotropic Kepler Problem", Mem. Am. Math. Soc. 52, 1-115 (1984)

${ }^{8}$ C. Vidal, "Periodic Solutions for Any Planar Symmetric Perturbation of the Kepler Problem", Cel. Mech., Dyn. Astr, 119-132, (80) (2001)

${ }^{9}$ G. Birkhoff, "The Restricted Problem of Three Bodies", Rend. Circ. Mat. Palermo, 39, 1-70 (1915)

${ }^{10}$ H. Poincaré, "Les Methods Nouvelles de la Mecanique Celeste", GauthierVillars (1892)

${ }^{11}$ V. Szebehely, Theory of Orbits (Academic Press, New York, 1967)

${ }^{12} \mathrm{~W}$. Thirring, A Course in Mathematical Physics I, Classical Dynamical Systems (Springer Verlag, New York, 1978)

${ }^{13}$ C. Stoica, "A proper set of orbital elements and perturbation equations in Maneff-type fields", preprint

${ }^{14}$ C. L. Siegel, Moser J.K., Lectures on Celestial Mechanics ( Springer-Verlag, Berlin, 1971) 